\title{
Impacto de la repatriación de utilidades de la IED estadounidense en América Latina*
}

\author{
Impact of Repatriation of US FDI Profits in Latin America \\ José Israel Briseño Perezyera, ** Miguel Ángel Martínez Garcia *** \\ y Omar Neme Castillo****
}

\section{RESUMEN}

Se estima el efecto de largo plazo de la repatriación de utilidades de la inversión extranjera directa estadounidense sobre un conjunto de economías latinoamericanas, siguiendo una metodología de cointegración de panel. Los resultados sugieren que la inversión en capital físico, empleo, gasto público en educación y la repatriación de utilidades de la IED impactan en la actividad económica de la región. En particular, la repatriación muestra signo negativo, constituyéndose en una barrera al crecimiento económico en la región. Por ende, una regulación que propicie competitivamente las reinversiones en las economías favorecería el desempeño económico en esos países.

Palabras clave: utilidades, reinversión, repatriación, América Latina, efectos económicos. Clasificación JEL: F21, O10, O40.

\begin{abstract}
We estimate the long-term effect of the repatriation of US FDI profits on a set of Latin American economies, following a panel cointegration metodology. The results suggest that investment in physical capital, labor, public expenditure on education and the repatriation of FDI profits impact on economic activity in the region. Particularly, repatriation shows a negative sign, becoming a barrier to economic growth. Thus, a regulation that competitively boosts the reinvestment would be favorable for economic performance in these countries.

Keywords: profits, reinvestment, repatriation, Latin America, economic effects. JEL classification: F21, O10, O40.

\footnotetext{
* Fecha de recepción: 06/05/2013. Fecha de aprobación: 25/05/2015.

** Profesor-investigador de la Sección de Estudios de Posgrado e Investigación, Escuela Superior de Economía del IPN. Correo electrónico: jobriper@hotmail.com.

*** Profesor-investigador de la Sección de Estudios de Posgrado e Investigación, Escuela Superior de Economía del IPN. Correo electrónico: mmartinezga@ipn.mx

**** Profesor-investigador de la Sección de Estudios de Posgrado e Investigación, Escuela Superior de Economía del IPN. Correo electrónico: oneme@ipn.mx.
} 


\section{INTRODUCCIÓN}

Las economías más dinámicas buscan crear las mejores condiciones en el mercado doméstico para atraer nuevas inversiones o reinversiones de los capitales extranjeros productivos, interesados en aumentar sus beneficios desde estos escenarios competitivos. Para la literatura empírica, la inversión extranjera directa (IED) condiciona el crecimiento económico de los países de destino, puesto que complementa aspectos del mercado interno como el empleo, capacidad de producción, flujos comerciales, actualización tecnológica, entre otros. ${ }^{1}$ En este contexto, los gobiernos de los países latinoamericanos, tradicionalmente receptores de este tipo de inversión, han implementado políticas activas para atraer estos capitales como alternativa hacia el desarrollo industrial y crecimiento económico.

A partir de la década de los ochenta, la política económica se orienta a la apertura e intensificación comercial y a la atracción de mayores flujos productivos desde el extranjero, en la mayoría de los países de América Latina, permitiendo una notable entrada de IED estadounidense hacia la región. De acuerdo con datos del Bureau of Economic Analysis (BEA) y de la Comisión Económica para América Latina y el Caribe (CEPAL), en 2013 el total de la inversión de Estados Unidos (EU) en América Latina fue de 38.2 miles de millones de dólares (equivalente a $6.9 \%$ del producto interno bruto (PIB) latinoamericano en ese año), con crecimiento promedio anual de $9.7 \%$. Esta cantidad es casi veinte veces mayor a la de 1980, cuando ese país invirtió 1.7 miles de millones de dólares (mmd).

Asimismo, las empresas transnacionales (ETN) estadounidenses alcanzaron importantes utilidades, con un notable avance (tasa de crecimiento promedio anual de $0.9 \%$ ) al pasar de $1.4 \mathrm{mmd}$ en 1980 a $24.6 \mathrm{mmd}$ en 2013 (representando $2.3 \%$ de la deuda pública de los países latinoamericanos considerados). En general, estas utilidades tienen dos destinos: reinversión en el mercado latinoamericano o repatriación a la economía de EU. En 1980, las utilidades repatriadas alcanzaron los 725 millones de dólares o $53 \%$ del total de las utilidades. Para 2013 fueron $9.5 \mathrm{mmd}$, o $39 \%$ del total de las utilidades, con un crecimiento promedio anual de $8.2 \%$. En otras palabras, la IED estadounidense en América Latina muestra una ligera tendencia a reinvertir, lo que concuerda con Ibarra-Caton y Mataloni (2014), UNCTAD (2013) y Higginbottom (2013), por ejemplo.

\footnotetext{
${ }^{1}$ Véase, por ejemplo, Fritsch y Franco (1991), y Pelegrín (2002).
} 
La reinversión de utilidades tiene efecto positivo en la economía receptora, por ser capital que se queda como nueva inversión, impulsando la dinámica de la misma. En contraste, la reinversión implica un efecto negativo para la economía de origen, dado que estos capitales marginales no se consolidan como nueva inversión en ésta, restringiendo las posibilidades de crecimiento del empleo, consumo, producción industrial e ingreso per cápita, entre otras variables.

Por el contrario, la repatriación de utilidades muestra dos efectos. El primero -negativo-, sobre el mercado latinoamericano al limitar directamente la disponibilidad doméstica de capitales y, por tanto, el ahorro e inversión, lo que a su vez, impacta negativamente en la generación de nuevos empleos, crecimiento económico e incluso en la recaudación tributaria futura. El segundo efecto -positivo- trae justamente estos beneficios para la economía estadounidense (Hines y Hubbard, 1990). ${ }^{2}$

No obstante, a pesar de la aparente relación entre repatriación (reinversión) de las utilidades de la IED y la actividad económica, la literatura empírica en este renglón es limitada. ${ }^{3}$ Brada y Tomsík (2009) hallan que la evolución de las utilidades y su destino generan distorsiones en ocho economías de Europa del Este, aceptando que la madurez de la IED en una economía es crucial en este proceso. Asimismo, Altzinger (2008) en un estudio para la Unión Europea, distingue entre IED nueva y las fusiones y adquisiciones, encontrando que durante los primeros años éstas son más rentables que las nuevas inversiones, por lo que tienen mayores tasas de repatriación, contribuyendo en menor medida al acervo de capital de la economía huésped al tiempo que mejora el desempeño económico del país de origen.

Para el caso de estudio, se aprecia una relación con mayores ventajas para EU. Esto es, la repatriación de utilidades a ese país parece beneficiar a dicha economía en términos de PIB, consumo final, PIB per cápita y, en menor medida, al em-

\footnotetext{
${ }^{2}$ Este efecto tiene una importancia adicional puesto que significa no sólo que las firmas en EU tienen acceso a esos capitales, sino también el gobierno de este país tasa las ganancias de las filiales estadounidenses hasta que son repatriadas.

${ }^{3}$ La mayoría de los estudios se centra en los efectos macroeconómicos de la IED, tanto en el país origen como en el receptor; muchos de ellos destacan el efecto en: 1) la balanza de pagos, por ejemplo: Mencinger (2003); Blomström y Kokko (2003); Borensztein et al. (1995); Lipsey (2002); Alfaro et al. (2004); Markusen y Venables (1999); 2) en los determinantes de la IED (macro-, micro- y específicos de las industrias), por ejemplo: Love y Lage (2000); Slaughter (2003); Kok y Acikgoz (2009); Chakrabarti (2001); Shantz y Venables (2000); 3) o en el efecto de los sistemas impositivos, en el país origen o anfitrión sobre las decisiones de reinversión o pago de dividendos; por ejemplo: Barry (2005); Gropp y Kostial (2000); Ishii (2006).
} 
pleo y la producción industrial. La repatriación aparentemente muestra una relación negativa con esas mismas variables para el caso latinoamericano. Así, se plantea la hipótesis que el aparente movimiento simultáneo entre repatriación y dichas variables, restringen la actividad económica en América Latina. Esto es, la presencia de ETN y las decisiones de reinversión, dada la competitividad de las economías latinoamericanas, tienden a limitar el crecimiento económico en la región.

En este sentido, el objetivo del trabajo es determinar para, un grupo de 14 países latinoamericanos, el efecto a largo plazo de la repatriación hacia EU de las utilidades resultantes de la IED localizada en aquéllos durante 1980-2013. Con esto, se pretende establecer, considerando sólo las utilidades de la IED, si en efecto es favorable la participación de esta inversión en la actividad económica en América Latina.

El documento se estructura en cinco secciones. En el primer apartado se realiza un breve recuento de los determinantes de la reinversión o repatriación. En la sección dos se revisa la literatura empírica del tema. En la tercera y la cuarta se presentan algunos hechos sobre América Latina y se plantea el modelo econométrico a estimar usando técnicas de cointegración de panel. En el apartado cinco se muestran los resultados para después concluir.

\section{REINVERSIÓN Y REPATRIACIÓN DE LAS UTILIDADES DE LA IED: ALGUNOS DETERMINANTES}

La reinversión de ganancias obtenidas por la IED impacta en los patrones de crecimiento de las economías, puesto que significa la retención de divisas para invertir productivamente en su territorio. En la medida que el acervo de IED madure, la posibilidad que nuevas inversiones se realicen en esa economía aumenta. Mientras más ETN operen en una economía, otras empresas son atraídas a invertir en ella, guiadas por decisiones estratégicas para competir a nivel mundial. En otras palabras, a mayor acumulación de IED en relación al tamaño de la economía y a mayor rentabilidad, mayor la magnitud de capitales que pueden reinvertirse en las filiales (Brada y Tomšik 2009).

De esta manera, distintos elementos influyen en la distribución de las utilidades de las filiales entre reinversión y repatriación. Uno de los factores determinantes son las oportunidades de inversión alternativas. De acuerdo con Lehmann (2002), una condición necesaria para la reinversión es la obtención de niveles positivos de utilidades en una industria en particular, que se entiende como una nueva oportunidad de inversión en la economía receptora, dadas las 
mejores condiciones de operación, lo que fomenta la reinversión de utilidades y la entrada de nuevos competidores.

Además, el problema de decisión entre estas dos opciones, repatriación o reinversión, implica la consideración de los rendimientos esperados a valor actual. Obersteiner (1973) identifica dos tipos de variables que afectan este valor. Por un lado, el sistema impositivo en las economías huésped y originaria. Por el otro, la incertidumbre económica en el país generador de las utilidades de esa inversión, donde sobresalen la inflación, el riesgo de devaluación, y riesgos políticos a los que se exponen las empresas transnacionales. ${ }^{4}$

La reinversión también depende de las diferencias en rentabilidad entre las filiales. En general, las diferencias de productividad y, por tanto, en la rentabilidad, reflejan los distintos niveles de eficiencia en el uso de capacidades y activos específicos a las matrices y a las filiales (Rugman y Verbeke, 2001). Así, en la medida que la rentabilidad resulte de la explotación de tales ventajas dentro de la economía receptora, se espera que ésta retenga los capitales mediante reinversión (Clausing, 2001).

Asimismo, una depreciación sostenida de la moneda del país receptor, tiende a desalentar la repatriación propiciando un incremento de la reinversión. Dunning (1993) considera al tipo de cambio como una variable con efecto crecimiento en la IED y no con efecto nivel. Para Lucas (1993), más que el tipo de cambio, lo que determina la repatriación es el riesgo cambiario.

Adicionalmente, los diferentes sistemas de gobierno corporativo influyen en esta decisión. Las empresas estadounidenses que operan en países con mercados de capitales relativamente desarrollados se orientan al desempeño financiero de corto plazo para aumentar el valor de los accionistas (Hall y Soskice, 2001), lo que implica mayores niveles de repatriación. Allen et al. (2000) afirman que los inversionistas prefieren comprar acciones de empresas que pagan regularmente dividendos.

Además, la repatriación depende de la estructura de propiedad de las filiales. Cuando la propiedad es parcial (o se localizan en lugares con débiles derechos de propiedad) las casas matrices está dispuestas a pagar mayores dividendos (Desai, Foley y Hines, 2002). Asimismo, el pago sistemático de dividendos es un

\footnotetext{
${ }^{4}$ Los modelos teóricos buscan identificar las variables clave que afectan la eficiencia de la asignación de recursos generados en el extranjero, entre usos alternativos, es decir, entre repatriación de dividendos a la casa matriz o reinversión de esos fondos en la economía donde se generaron. En concreto, las aportaciones teóricas miden los rendimientos comparativos de las alternativas dadas ciertas restricciones internas (gobierno corporativo) y externas (factores económicos).
} 
mecanismo de monitoreo y disciplina de los directivos en las filiales extranjeras (Lehmann y Mody, 2004). En un escenario en el que el objetivo de los directivos diverge al de los accionistas surgen costos de agencia. Una forma de reducir estos costos es a través del pago regular de dividendos que redistribuye los rendimientos de la inversión y reduce las opciones de comportamientos oportunistas.

Lehmann y Mody (2004) profundizan en el rol de los dividendos y examinan la tasa de pago, es decir, el cociente entre el pago de dividendos y las utilidades netas obtenidas en el país receptor. Señalan que el pago sistemático de dividendos lleva a una volatilidad en la reinversión de beneficios en la economía anfitriona. Por tanto, el patrón de repatriación tiene implicaciones en el volumen y estabilidad de la IED.

Por último, una variable central para la reinversión son los impuestos sobre el ingreso de las filiales. Un cambio en los impuestos aplicables a las filiales modifica los incentivos y, por tanto, las decisiones. La tasa impositiva sobre los ingresos de ETN que envían ganancias desde sus filiales es diferente en cada país (Lundan, 2006). ${ }^{5}$ Así Desai, Foley y Hines (2001) señalan que existe una correlación positiva entre tasas impositivas y utilidades repatriadas.

Las diferencias de tasas impositivas a las utilidades entre el país anfitrión y el receptor, afectan la conveniencia de reinvertir dadas las condiciones económicas de las casas matrices. Los fondos tienden a invertirse en los mercados con mayores beneficios fiscales. Dado que las casas matrices tienen que pagar impuestos, los incentivos para aplazar la repatriación son mayores para las filiales en países con bajos impuestos (Desai, Foley y Hines, 2002).

Por último, existen factores contables que influyen en la decisión sobre el uso de estas utilidades. Para Graham, Hanlon y Shevlin (2011), el diferimiento del pago de impuestos a las ganancias vía mecanismos contable-financieros es vital para la ubicación de las operaciones y la posibilidad de repatriar o reinvertir; lo que se acentúa en el caso de empresas que cotizan en bolsa, con activos en el extranjero y altos gastos en investigación y desarrollo.

\section{REVISIÓN DE LA LITERATURA}

En el caso de la región latinoamericana, existe poca evidencia empírica de los efectos de la repatriación. No obstante, algunas investigaciones concuerdan que

\footnotetext{
${ }^{5}$ Los impuestos sobre las filiales generan efectos de ubicación, afectando la forma en que las ETN eligen repatriar sus ganancias, ya sea a través de dividendos intrafirma, pagos de intereses o de regalías, además de precios de transferencia.
} 
la repatriación tiende a generar efectos adversos en estos países. Así, la reinversión de utilidades se ha convertido en un componente crucial de la IED en la región, ${ }^{6}$ incluso el crecimiento de las reinversiones ha disminuido la proporción de nueva IED (Higginbottom, 2013).

Kliman (2012) reconoce la reinversión de utilidades de la IED como un componente de la acumulación de capital no productivo de las ETN estadounidenses. Encuentra que sólo una parte de la reinversión se usa en la compra de activos fijos (estructuras, equipo, software, etc.), mientras que otra parte se destina a la inversión en instrumentos financieros o, incluso, se mantiene en efectivo (Blouin et al., 2012). Aún más, atribuye la totalidad del crecimiento en la tasa de reinversión al crecimiento de este tipo de capital no productivo.

Así, la reinversión de transnacionales de Eu ha llevado a una acumulación de activos en América Latina, lo que en palabras de Higginbottom (2013) implica "la construcción de una presencia económica permanente que le otorga derechos respecto a los beneficios futuros y poder político". Esto contribuye al proceso de desnacionalización, descapitalización y dependencia de las estrategias de las ETN.

Al respecto, Mortimore (2006) afirma que la participación de ETN en la región se concentra en cuatro tipos de estrategias de búsqueda: 1) de materias primas, 2) de mercados locales (nacional o regional), 3) de eficiencia para el acceso a mercados y 4) de activos tecnológicos. Estas estrategias tienden a generar efectos negativos como actividades de enclave que llevan a desarticulaciones productivas, baja competitividad a nivel mundial, escaso procesamiento local de recursos, contaminación ambiental, limitado desarrollo local de la producción y de servicios, bajo valor agregado, desplazamiento de empresas locales, aumento de incentivos fiscales a empresas extranjeras, baja propensión a la inversión tecnológica, bajo o nulo desarrollo científico y tecnológico, entre otros. ${ }^{7}$

Por otro lado, Agostini y Jalile (2009) destacan que 11 países de América Latina tienden a disminuir las tasas de impuestos a las utilidades de la IED, tratando de captar más de ésta. También establecen que la elasticidad impuesto de la IED oscila entre 0.75 y 0.96 . Sin embargo, una proporción importante sólo se mantiene en cuentas bancarias (Blouin et al., 2012). Asimismo, Echavarría (2005) determina que el impuesto a las utilidades de las empresas es altamente

\footnotetext{
${ }^{6}$ La UNCTAD (2012) señala que en 2003-2011, 45\% de los flujos de IED fueron de reinversión.

${ }^{7}$ En particular, para el caso mexicano, Carrillo y Gomis (2011) dimensionan el problema al señalar que 58\% de las ETN siguen la estrategia de búsqueda de eficiencia en mercados externos, 34\% busca el mercado regional o nacional y $7.7 \%$ busca activos tecnológicos.
} 
distorsionante, complejo y con efectos ocultos en el caso concreto de Colombia. Igualmente, señala que las bajas tasas de impuestos atraen IED, desincentivan la evasión y elusión, e incrementan la recaudación fiscal, mientras que las altas tasas tienen el efecto contrario.

\section{Algunos heChos: IED EN AMÉRICA LATINA}

Dado el agotamiento del modelo de sustitución de importaciones, las economías de la región reorientaron sus estrategias de industrialización, estableciendo un modelo de desarrollo orientado al exterior, con la intención de mejorar la eficiencia económica, reducir la intervención estatal y propiciar la integración en la economía internacional. Como consecuencia, en 2013, el comercio internacional y la entrada de IED de entrada en la región fueron poco más de diez veces mayores a los de 1985. Al respecto, EU es una de las principales fuentes de IED para América Latina, con un promedio de 30\% del total en el periodo. No obstante, esta proporción se redujo de 38\% en 1985 a 24\% en el último año (CEPAL, 2014). Esto sugiere que las firmas estadounidenses han consolidado sus sistemas de producción en la región y, al mismo tiempo, enfrentan una mayor competencia principalmente de países europeos y asiáticos. ${ }^{8}$

Esta tendencia en la atracción de capitales estadounidenses a la economía latinoamericana se debe a que ciertas restricciones a estas inversiones sólo se han relajado gradualmente y de forma no discriminatoria para otros países industrializados, por lo que este mecanismo no representó gran impulso en la atracción de firmas de EU, comparado con las entradas de IED desde terceras economías (Morales, 2010).

En este contexto, los marcos institucionales de los países han reorientado las políticas sobre inversión extranjera, laboral y tributaria tratando de incentivar nuevos flujos de IED y su permanencia dentro de las economías individuales. Así, de acuerdo con Morales, Guerrero y López (2009), en los últimos veinte años la flexibilización de los marcos regulatorios de estos países, además, de la estabilidad macroeconómica, permitieron un mayor ingreso de estos capitales.

Adicionalmente, el desempeño de la IED y, por ende, de la reinversión, está restringido por el Acuerdo sobre las Medidas en materia de Inversiones relacionadas con el Comercio (AMIC), que prohíbe el uso de medidas relacionadas

\footnotetext{
${ }^{8}$ De acuerdo con la CEPAL (2014), la Unión Europea, en conjunto, constituye la mayor fuente de IED destinada a la región en 2013.
} 
con el comercio como requisitos de contenido local (Mortimore, 2009). Las inversiones extranjeras han fluido como resultado de procesos de privatización, apertura comercial y reducción de restricciones sobre la IED respecto a la repatriación de utilidades, restricciones sectoriales (contenido local y requisitos de exportación) y autorización previa para invertir (Blomström y Wolff, 1994).

Asimismo, Gligo (2007) advierte que los países de América Latina han seguido un marco de políticas activas caracterizado por dos elementos: políticas de promoción (particularmente, las agencias de promoción de inversiones, API) y políticas de incentivos. Respecto a las primeras, se señala que treinta países latinoamericanos cuentan con una API. Éstas han captado nuevas inversiones y generado reinversiones mediante servicios postinversión (after-care).

En cuanto a las segundas, se tiene que los incentivos en general se agrupan en fiscales (rebajas temporales o permanentes de impuestos), financieros (subsidios directos que reducen el costo de operación de la inversión) o jurídicos (garantías establecidas en acuerdos bilaterales). De igual manera, el incentivo principal es de tipo fiscal, orientado a la instalación de empresas, en zonas francas con exenciones al impuesto, que buscan incrementar la eficiencia y participan en mercados de exportación.

Un aspecto concreto respecto a las políticas de incentivos son los llamados Acuerdos para la Promoción y Protección Recíproca de las Inversiones (APPRIS). La mayoría de los países latinoamericanos han establecido este tipo de mecanismo con la intención de promover y proteger las inversiones y atraer, en consecuencia, capitales extranjeros. Los APPRIS ofrecen al inversor extranjero garantías para sus capitales en aspectos como trato justo y equitativo, a través de cláusulas de Trato Nacional y de Nación Más Favorecida, además del derecho a indemnización ante expropiaciones, así como la libre transferencia de las utilidades obtenidas.

Granato y Oddone (2007) señalan que un punto clave de los APPRIS es la cláusula de libre transferencia de divisas, en la que se da seguridad jurídica para que los inversores extranjeros transfieran libremente y sin restricciones todos los pagos relacionados con sus inversiones, incluida la ganancia del capital invertido y el producto de la liquidación total o parcial del mismo. Adicionalmente, estos acuerdos tienden a prohibir requisitos de desempeño, impidiendo que el país anfitrión establezca medidas protectoras de la industria nacional como condición para las inversiones extranjeras.

En el caso particular de los países, para México se tiene, por ejemplo, que en 1989 se estableció un nuevo mecanismo de impulso a la IED (el Entendi- 
miento para Facilitar el Comercio y la Inversión) que eliminó o redujo obstáculos (regulaciones de contenido doméstico) (Dussel, Galindo y Loría, 2003). Asimismo, en la Ley de Inversión Extranjera Directa de 1993, los requisitos de desempeño de estas inversiones se eliminaron al tiempo que no se establecieron restricciones sectoriales. ${ }^{9}$

En Chile, el marco regulatorio de la IED (Decreto Ley 600 o Estatuto de la Inversión Extranjera, DL600) de 1974, ha sufrido cambios para generar certidumbre en las inversiones. El DL600 se basa en los principios de trato nacional y no discriminación como mecanismos para atraer y promover inversiones, nacionales y extranjeras, por lo que sujeta a la IED al régimen jurídico aplicable a la inversión nacional. Dentro de este régimen, los inversores pueden transferir al exterior sus capitales y las utilidades líquidas obtenidas, aunque las remesas de capital sólo pueden realizarse después de un año de su ingreso (Rodas, 2005).

Asimismo, Costa Rica modificó su estrategia de atracción de capitales extranjeros y creó un régimen especial para la IED vinculado a las exportaciones. Los incentivos son de naturaleza fiscal, establecidos en leyes que promueven las zonas francas. En esta nueva estrategia, se tienen regímenes impositivos especiales y para reexportación para las empresas en las Zonas Especiales para el Procesamiento de Exportaciones.

Por último, en 1990, Brasil aprobó el Programa Nacional de Desestatización, que se concentró en la venta de empresas estatales estratégicas (siderúrgicas, petroquímicas y fertilizantes). Estableció una serie de reformas constitucionales, para facilitar la entrada del capital extranjero, y redefinió el concepto de empresa brasileña, contando con una ley general que regula el régimen de la IED, además de regulaciones sectoriales específicas. Los principios que regulan la IED son: ausencia de autorización previa, igualdad de condiciones para el capital nacional y extranjero, libre inversión a través de filiales o joint ventures y libre repatriación del capital. Los incentivos se basan en beneficios fiscales, financiamiento específico y programas de apoyo, entre otras.

Así, en un contexto de economía cerrada que caracterizó a América Latina hasta la década de los ochenta, el flujo de capitales productivos, particularmente desde EU, se mantuvo en niveles relativamente estables. Dadas las restricciones normativas que regulaban la IED en actividades productivas dentro

\footnotetext{
${ }^{9}$ No obstante, dentro del TLCAN existen diferentes regulaciones respecto a las reglas de origen para sectores específicos que promueven la integración entre las tres economías pertenecientes a este acuerdo comercial.
} 
de la región, se generó, en términos de Dussel (1997), una coexistencia pacífica entre empresas nacionales y extranjeras que llevó a que las filiales mantuvieran tasas de utilidades comparativamente bajas en 1980-1993 de apenas 0.28\% del PIB en promedio.

No obstante, en ese periodo se registró una ligera tendencia a repatriar; las utilidades enviadas a la economía de EU representaron $0.19 \%$ del PIB, impactando en última instancia la entrada neta de IED. En contraste, con el mayor intercambio comercial con la región, las ETN estadounidenses renovaron sus estrategias empresariales materializándose en mayor IED en la región en los últimos años (América Latina es el principal destino para las inversiones estadounidenses entre las regiones en desarrollo). Las regulaciones sobre este tipo de inversiones, en las que se incluye la reinversión de utilidades, tuvo como consecuencia un aumento en la tasa de utilidades que alcanzó $0.58 \%$ del PIB, aunque la tendencia, como proporción del PIB, aumentó durante 1994-2013 a 0.35\%.

\section{Modelo, datos Y Metodología}

Dado que el interés es determinar el efecto de la repatriación, que en última instancia son capitales para la inversión, sobre la expansión de la actividad económica, la teoría económica subyacente que se emplea es la de crecimiento endógeno. En particular, se especifica un modelo partiendo de la función de producción neoclásica de cambio tecnológico endógeno de Romer (1990), esto es:

$$
Y=K^{\alpha}(A H)^{\beta} T^{\gamma}(A L)^{1-\alpha-\beta-\gamma}
$$

Asumiendo una relación lineal logarítmica entre las variables repatriación de utilidades de la IED de EU desde América Latina (reuied), capital físico $(f b k f)$, trabajo (pea) y capital humano (gpepib), la especificación del modelo, que se estima en la siguiente sección siguiendo un análisis de cointegración de panel, es: ${ }^{10}$

\footnotetext{
${ }^{10}$ Inicialmente se consideraron dos variables explicativas adicionales definidas como: 1) bcmanuf, apertura comercial que lleva a los países a importar bienes de mayor calidad sin que se produzcan localmente, generando un efecto positivo en el PIB vía aumento de las tasas de aprendizaje, disponibilidad de innovaciones, mayor uso de bienes de capital avanzado, etc. Se emplea como medida indirecta la balanza comercial manufacturera en dólares constantes. La base de datos es la UN Comtrade Data Base de la ONU. El signo esperado es positivo; y 2) gidpib: innovación tecnológica, puesto que el nivel de producto depende positivamente del acceso y uso de tecnologías avan-
} 
aeit $=\beta_{i 1}+\beta_{i 2}{ }_{\text {reuied }}{ }_{i t}+\beta_{i 3}{ }_{i b}^{\text {fblf }}+\beta_{i 4}{ }_{i t}^{\text {pea }}+\beta_{i 5}{ }_{\text {gpegpt }}+\beta_{i t} 6^{\text {gidpib }}{ }_{i t}+\beta_{i} 7^{\text {bcmanuf }}{ }_{i t}+u_{i t}$

donde $i$ : un país de América Latina. El estudio se centra en 14 países para el periodo 1980-2013, dada la disponibilidad de datos, ${ }^{11}$ lo que da un total de 476 observaciones. Todas las variables se expresan en logaritmos para incluir los efectos multiplicativos de las series de tiempo. Si estas variables comparten una tendencia estocástica común y sus primeras diferencias son estacionarias, entonces existe al menos una relación de equilibrio de largo plazo.

Se estima un modelo que incluye, además de la repatriación de utilidades, variables macroeconómicas para considerar las elasticidades en el PIB, consumo, producción industrial e ingreso per cápita, entre éstas:

$a e$ : actividad económica. Se espera que las variables explicativas impacten en la economía del país receptor. Se emplean diversas medidas, por lo que se establecen cuatro especificaciones distintas de la relación; que incluyen: 1) tcpib, para determinar el efecto de reuied en el crecimiento económico; 2) $c f$, que representa la tasa de crecimiento del consumo final; 3) pibpc, para capturar el efecto de esta repatriación en la senda de desarrollo de las economías, y 4) ipi, entendida como el índice de la producción industrial y que se incluye para establecer el "financiamiento" de las ganancias de la IED a la actividad manufacturera. Los datos de ésta se toman de la oficina de estadísticas de cada país de América Latina y de la UN Stats (National Accounts Main Aggregates Database). Las series de las tres primeras variables se obtienen de la UN Stats. Se espera que la relación entre éstas y reuied sea negativa. Según el caso, las series aparecen en dólares constantes.

reuied: repatriación de utilidades de la IED estadounidense desde América Latina. Dado que la actividad económica de un país depende del nivel de inversión, nacional o extranjera, se espera que la repatriación signifique menor disponibilidad

zadas, se tiene que a mayor acervo de tecnología, mayor tasa de crecimiento. Se emplea el gasto en investigación y desarrollo como porcentaje del PIB, en dólares constantes. Las series se toman de la OECD Stats. Sin embargo, dado que el orden de integración de bcmauf y gidpib es distinto al del resto de las variables, de acuerdo con las pruebas de raíz unitaria, en el análisis de cointegración no se consideran estas dos variables.

${ }^{11}$ Las economías para las que existían datos completos en el periodo son: Argentina, Bolivia, Brasil, Chile, Colombia, Costa Rica, Ecuador, Honduras, México, Panamá, Paraguay, Perú, Uruguay y Venezuela. 
de capitales para su uso productivo. Por tanto, el signo esperado es negativo. Las series se obtienen de la base International Economic Accounts del Bureau of Economic Analysis (BEA) y se expresan en dólares constantes.

$f b k f$ : capital físico. Se asume que existe una relación directa entre el acervo de capital y la producción reflejada en la productividad marginal de este factor. A mayor inversión en la formación de capital físico de origen nacional, mayor el nivel obtenido de producto, por lo que el signo esperado es positivo. Para corrobóralo, se emplea la formación bruta de capital fijo, en dólares constantes. La fuente es la UN Stats.

pea: mano de obra, calificada o no. Dado que la productividad marginal de este factor se relaciona con el crecimiento en la economía, se espera un signo positivo en el coeficiente de esta variable que se aproxima a través de la población económicamente activa. Los datos se toman de la LaborStat de la Organización Mundial del Trabajo y de las oficinas respectivas de cada país de América Latina.

gpegpt: capital humano. El nivel de producto se determina crecientemente por la disponibilidad y calidad del capital humano, puesto que estos factores generan rendimientos no decrecientes en la producción. Por ende, cuanto mayor acervo de trabajo capacitado tenga un país, mayor la tasa de crecimiento, esperándose un signo positivo. La medida indirecta empleada es el gasto en educación como porcentaje del gasto público total, en dólares constantes. Las series se toman del Instituto de Estadísticas de la UNESCO.

Se emplean técnicas de cointegración de panel para estimar el efecto de la repatriación en la actividad económica de distintos países de América Latina, que permite usar toda la información disponible, lo cual no es detectable en las estimaciones de corte transversal y series de tiempo de forma separada. La metodología de datos de panel incrementa el poder de las pruebas cuando la muestra disponible de datos es pequeña, al combinar elementos de datos de panel con series de tiempo.

En este tipo de metodología es requisito determinar el nivel de integración de las series para evitar resultados espurios. Dado que los datos de panel incrementan el poder de las pruebas al ampliar la dimensión de las series por las secciones cruzadas, los resultados son más confiables que las pruebas estándar, como la de Dickey-Fuller aumentada. Existen dos tipos de pruebas de raíz unita- 
ria para datos de panel: procesos de raíz unitaria común, que se presenta cuando las unidades de sección cruzada comparten parámetros entre sí (Levin, Lin y Chu, prueba LLC), y procesos de raíz unitaria individual, cuando los parámetros son diferentes entre unidades (Im, Pesaran y Shin, prueba IPS).

Mientras que la prueba LLC permite heterogeneidad de los efectos determinísticos individuales y de la estructura de correlación serial, supone la hipótesis alternativa de presencia de raíces autorregresivas homogéneas (mismo coeficiente del término autorregresivo), lo que limita su poder. La prueba evalúa la hipótesis de no estacionariedad de cada serie individual mediante estadísticos $t$ agrupados.

La prueba IPS supera la limitación de la LLC suponiendo que existe heterogeneidad de la raíz autorregresiva. Esta prueba calcula la correlación serial residual, permitiendo distintos coeficientes de los términos autorrregresivos, basada en el promedio simple de los estadísticos Dickey-Fuller (DF) de cada sección cruzada en el panel. ${ }^{12}$

Una alternativa son las pruebas tipo Fisher, que, primero, prueban la existencia de raíces unitarias para cada panel individualmente y, después, combinan los valores de $p$ resultantes para la prueba total. De acuerdo con Choi (2001) estas pruebas eliminan los problemas en la LLC y la IPs. Por ende, a manera de análisis de robustez, se emplean las pruebas de raíz unitaria de panel LLC, IPS, ADF-Fisher y PP-Fisher. ${ }^{13}$ Para las dos primeras, la longitud de retardo óptima se determina de acuerdo con el criterio de Schwarz.

De este modo, si el proceso generador de datos de las variables se caracteriza por raíces unitarias, el siguiente paso es probar la existencia de relaciones estables de largo plazo desde una perspectiva de panel, para lo que existen dos enfoques. La metodología de Pedroni, McCoskey y Kao y Kao consiste en prue-

${ }^{12}$ No obstante, las simulaciones indican que la prueba IPs es sensible al número de rezagos en las regresiones DF. Asimismo, el poder de la prueba IPS es mayor cuanto más grande sea la dimensión de las unidades de sección cruzada. Además, la interpretación de los resultados de esta prueba es difícil dada la heterogeneidad subyacente en la hipótesis alternativa (Chandra y Sahoo, 2007).

${ }^{13}$ Se aplica una prueba de raíz unitaria a cada país individualmente y, después se combinan los valores de $p$ para construir el estadístico Fisher para determinar si la serie exhibe una raíz unitaria. Como la especificación de la prueba es: $\Delta \mathrm{y}_{i t}=\rho y_{i t-1}+z_{i t}^{\prime} \gamma_{i}+v_{i t}$; donde $i=1, \ldots, N, t=1, \ldots, T, v_{i t}$ es el término de error estacionario del $i$-ésimo país en el periodo $t$, respectivamente; $y_{i t}$ es la variable de estudio; $z_{i t}^{\prime}$ representa las variables de control (incluyendo repatriación), la $\mathrm{H}_{0}: \rho_{i}=1$ para toda $i$, $\mathrm{y}$ la $\mathrm{H}_{\mathrm{a}}<0$ para alguna $i$. Choi (2001) propone cuatro métodos de prueba de raíz unitaria en función del tipo de transformación realizada a los valores de $p: 1) \chi^{2}$ inversa $\left.(P), 2\right)$ normal inversa $\left.(Z), 3\right)$ logit inversa $(L)$ y 4$)$ modificación del método $\chi^{2}$ inversa empleado en muestra grandes. Para Breitung (2005), el estadístico $Z$ tiene una mejor relación de tamaño y robustez. 
bas residuales basadas en el procedimiento de Engle y Granger (EG), en el que la hipótesis nula de no cointegración se prueba usando los residuos de la regresión de panel. ${ }^{14}$ Por su parte, las pruebas de Groen y Kleibergen y Larsson, Lyhagen y Löthgren siguen un enfoque de máxima verosimilitud. ${ }^{15}$

Siguiendo a Pedroni (1995), es necesario emplear un procedimiento de determinación de cointegración de panel robusto en presencia de heterogeneidad. Al respecto, la prueba de Pedroni (1999) considera la heterogeneidad incorporando parámetros específicos que pueden variar entre los países de la muestra, lo que representa una ventaja, dado que asumir que todos los vectores de cointegración son idénticos para todos los elementos es poco real.

Esta prueba amplía la metodología residual de dos pasos de EG que analiza los residuales de la regresión espuria con variables estacionarias en primeras diferencias. Para que exista cointegración se requiere que $\varepsilon_{i t} \mathrm{I}(0)$. Formalmente, la prueba de Pedroni es:

$$
y_{i t}=\alpha_{i}+\beta_{1 i} x_{1 i t}+\cdots+\beta_{m i} x_{m i t}+\varepsilon_{i t}
$$

donde: $i=1, \ldots, N, t=1, \ldots, T, m=1, \ldots, M ; \alpha_{t}$ es un intercepto específico a cada país y $m$ el número de regresores. Se asume que $y$ y $x$ tienen orden de integración uno. Los coeficientes $\beta_{i}$ varían entre cada país. Para estimar los residuales de la ecuación (3), Pedroni (1999) propone siete estadísticos, cuatro basados en una dimensión intragrupos y tres en una dimensión entre grupos, a partir de la hipótesis nula de no cointegración. Sugiere ajustes sobre cada uno de los estadísticos tal que puedan compararse con la distribución normal estándar. Los resultados reportados en este documento se ajustan de esa manera.

Una vez que se determina la cointegración de panel, se estima la ecuación (3). De acuerdo con Pedroni (2000), la metodología de mínimos cuadrados ordinarios completamente modificados (FMOLS, por sus siglas en inglés) genera resultados más robustos cuando se analiza un panel heterogéneo con variables I(1); su distribución es estándar, asintóticamente no sesgada y libre de parámetros de perturbación. Este estimador emplea una corrección semiparamétrica para eliminar problemas causados por la correlación de largo plazo entre el vector de cointegración y las innovaciones en los regresores (Phillpis y Hansen, 1990). Así, el estimador FMOLS produce errores estándar y estadísticos $t$ consistentes.

${ }^{14}$ Véase Pedroni (1997), Pedroni (1999), McCoskey y Kao (1998), Kao (1999) y Engle y Granger (1987).

${ }^{15}$ Véase Groen y Kleibergen (2003) y Larsson, Lyhagen y Löthgren (2001). 
Asimismo, la prueba de Pedroni tiene dos especificaciones de cointegración heterogénea y homogénea. En la primera, el estimador se basa en la dimensión temporal del panel que permite obtener estimaciones de la relación de largo plazo, para cada sección cruzada en el panel (within dimension). En la segunda, el estimador considera la dimensión de corte transversal en el panel (between dimension). En este caso, las estimaciones representan el valor medio de los vectores de cointegración, es decir, los vectores son idénticos para todas las unidades en el panel. La mayoría de las especificaciones empíricas son de cointegración homogénea, lo que parece plausible, incluso para el actual análisis. Sin embargo, esta estructura es demasiado restrictiva (Breitung y Pesaran, 2005); asimismo, permitir que todos los parámetros sean específicos para cada unidad, reduce el sentido mismo del panel. Por tanto, parece más importante identificar parámetros probablemente similares para el conjunto de países estudiados, al tiempo que se permite cierto grado de heterogeneidad. No obstante, en este trabajo se siguen las dos especificaciones en la estimación.

Por último, se emplea el modelo de vectores de corrección del error (VECM) para detectar, desde un enfoque alternativo, el sentido de la causalidad implícita en la relación de cointegración. Todas las variables en el VECM son consideradas endógenas, por lo que el sistema de ecuaciones puede representarse:

$$
\Delta y_{i t}=\alpha_{i}+\sum_{j=1}^{p} \gamma_{i j} \Delta y_{i t-1}+\sum_{j=1}^{p} \gamma_{i j} \Delta x_{i j t-1}+\cdots+\sum_{j=1}^{p} \gamma_{m j} \Delta x_{m j t-1}+\omega_{i} t c e_{t=1}+u_{i t}
$$

donde $u_{i t}$ son los errores distribuidos normal y los tce, los términos de corrección del error rezagados que miden el ajuste al equilibrio de largo plazo; así, los coeficientes $\omega_{i}$ representan las correcciones de las variables hacia el equilibrio de largo plazo. Además, la causalidad de corto plazo existe si los $\gamma_{i}$ de las variables independientes son significativos, indicando que la variable dependiente responde a las desviaciones del equilibrio.

\section{Repatriación deSde AmÉrica Latina. Algunos resultados}

Para checar la estacionariedad de las series, se emplean dos tipos de pruebas de raíz unitaria de panel. En un proceso de raíz unitaria común se sigue la prueba LLC y en un proceso de raíz unitaria individual se usan los test IPS, ADF-Fisher y PP-Fisher. Los resultados de las pruebas en niveles y primeras diferencias se muestran en el cuadro 1. La hipótesis nula de raíz unitaria puede rechazarse para 
la diferencia del logaritmo de todas las variables, tanto para las pruebas con procesos comunes como individuales.

Cuadro 1. Pruebas de raíz unitaria

\begin{tabular}{|c|c|c|c|c|}
\hline \multirow{2}{*}{ Variable } & LLC & IPS & ADF-F & PP-F \\
\hline & Valor de $p$ & Valor de $p$ & Valor de $p$ & Valor de $p$ \\
\hline pib & 0.8932 & 0.9326 & 0.9975 & 0.9569 \\
\hline$\Delta p i b$ & 0.0000 & 0.0000 & 0.0000 & 0.0000 \\
\hline pibpc & 0.9921 & 0.9775 & 0.9563 & 0.9356 \\
\hline$\Delta p i b p c$ & 0.0000 & 0.0000 & 0.0000 & 0.0000 \\
\hline$c f$ & 0.9756 & 0.9985 & 0.9964 & 0.9562 \\
\hline$\Delta c f$ & 0.0000 & 0.0000 & 0.0000 & 0.0000 \\
\hline ipi & 0.9732 & 1.0000 & 0.9754 & 0.9874 \\
\hline$\Delta i p i$ & 0.0000 & 0.0000 & 0.0000 & 0.0000 \\
\hline reuied & 0.9216 & 1.0000 & 1.0000 & 0.9770 \\
\hline$\Delta$ reuied & 0.0000 & 0.0000 & 0.0000 & 0.0000 \\
\hline$f b k f$ & 0.9089 & 0.9055 & 1.0000 & 0.9562 \\
\hline$\Delta f b k f$ & 0.0000 & 0.0000 & 0.0000 & 0.0000 \\
\hline I & 0.9215 & 0.9823 & 0.9563 & 0.2347 \\
\hline$\Delta l$ & 0.0000 & 0.0000 & 0.0000 & 0.0000 \\
\hline gpepib & 0.7533 & 0.8905 & 0.9954 & 0.9963 \\
\hline$\Delta$ gpepib & 0.0000 & 0.0000 & 0.0000 & 0.0000 \\
\hline gidpib & 0.0256 & 0.0356 & 0.0256 & 0.0341 \\
\hline$\Delta$ gidpib & - & - & - & - \\
\hline $\mathrm{bcm}$ & 0.0416 & 0.0086 & 0.0454 & 0.0563 \\
\hline$\Delta b c m$ & - & - & - & - \\
\hline
\end{tabular}

$\mathrm{H}_{0}$ : raíz unitaria. La prueba LLC supone un proceso de raíz unitaria común. Las pruebas IPS, LADF-F y PP-F suponen un proceso de raíz unitaria individual. Significación al $1 \%$.

Las probabilidades de las pruebas Fisher se obtienen usando una distribución asintótica $\chi^{2}$. El resto de las pruebas asumen una distribución asintóticamente normal.

Dado que las variables integradas son del mismo orden, en este caso $I(1)$, se facilita el examen de las posibles relaciones de largo plazo mediante las pruebas de cointegración de panel de Pedroni. Los resultados de estos test se muestran en el cuadro 2. La hipótesis nula de no cointegración se rechaza para la mayoría de las pruebas en todas las ecuaciones. Los estadísticos panel-ADF y group-ADF son significativos al 5\%. Además, los estadísticos panel y group rho y 
PP son significativos. Así, los resultados evidencian que la repatriación de utilidades y otras variables relevantes se cointegran.

Cuadro 2. Prueba de cointegración de panel de Pedroni

\begin{tabular}{|c|c|c|c|c|c|c|c|}
\hline Ecuación & $\begin{array}{c}\text { Panel } \\
\text { v-Statistic }\end{array}$ & $\begin{array}{c}\text { Panel } \\
\text { rho- } \\
\text { Statistic }\end{array}$ & $\begin{array}{c}\text { Panel } \\
\text { PP- } \\
\text { Statistic }\end{array}$ & $\begin{array}{c}\text { Panel } \\
\text { ADF- } \\
\text { Statistic }\end{array}$ & $\begin{array}{c}\text { Group } \\
\text { rho- } \\
\text { Statistic }\end{array}$ & $\begin{array}{c}\text { Group } \\
\text { PP- } \\
\text { Statistic }\end{array}$ & $\begin{array}{c}\text { Group } \\
\text { ADF- } \\
\text { Statistic }\end{array}$ \\
\hline $\begin{array}{l}\text { dlpib dlreuied dlfbkf } \\
\text { dll dlgpepib }\end{array}$ & -2.533 & -2.941 & -6.515 & -2.065 & -1.397 & -6.275 & -2.944 \\
\hline prob & 0.975 & 0.004 & 0.000 & 0.039 & 0.044 & 0.002 & 0.019 \\
\hline $\begin{array}{l}\text { dlpibpc dlreuied } \\
\text { dlfbkf dll dlgpepib }\end{array}$ & -2.736 & -2.946 & -6.131 & -2.543 & -2.778 & -8.752 & -2.895 \\
\hline prob & 0.993 & 0.009 & 0.000 & 0.012 & 0.014 & 0.000 & 0.002 \\
\hline $\begin{array}{l}\text { dlcf dlreuied dlfbkf } \\
\text { dll dlgpepib }\end{array}$ & -2.940 & -3.134 & -8.112 & -2.709 & -2.958 & -8.054 & -3.875 \\
\hline prob & 0.905 & 0.002 & 0.000 & 0.004 & 0.007 & 0.002 & 0.000 \\
\hline $\begin{array}{l}\text { dlipi dlreuied dlfbkf } \\
\text { dll dlgpepib }\end{array}$ & -3.025 & -3.461 & -9.260 & -5.244 & -3.131 & -11.788 & -4.596 \\
\hline prob & 0.902 & 0.000 & 0.000 & 0.000 & 0.006 & 0.000 & 0.000 \\
\hline
\end{tabular}

$\mathrm{H}_{0}$ : no cointegración

Dada la evidencia de cointegración en el panel, se estiman los parámetros de interés, esto es, el impacto de largo plazo de la repatriación de utilidades y las variables de control en la actividad económica en distintos países de Latinoamérica, usando el estimador FMOLs. Esta elección, frente al estimador de mínimos cuadrados ordinarios (MCO), se basa en que FMOLS corrige problemas de correlación serial y de endogeneidad que pueden surgir con MCO (Acharya, Amanulla y Joy, 2009). El cuadro 3 presenta los resultados de las estimaciones para las cuatro ecuaciones.

En general, se encuentran los signos esperados. Específicamente, los resultados indican que la inversión en capital físico, el personal empleado y la formación de capital humano, tienen impacto estadísticamente positivo en el largo plazo sobre la actividad económica ( $p i b$ y $c f$ ), productiva (ipi) y en los niveles de desarrollo (pibpc). La significación de la variable capital humano (gpegpt) sugiere la validez del argumento neoclásico respecto al rol clave de esta variable para el crecimiento económico. 
Cuadro 3. Estimaciones mediante FMOLS* (panel group)

\begin{tabular}{|c|c|c|c|c|}
\hline \multirow{2}{*}{$\begin{array}{c}\text { Variables } \\
\text { independientes }\end{array}$} & \multicolumn{4}{|c|}{ Variables dependientes } \\
\hline & dlpib & dlpibpc & $d / c f$ & dlipi \\
\hline dlreuied & -0.029 & -0.021 & -0.040 & -0.032 \\
\hline$t$ & -2.08 & -2.35 & -2.09 & $-2.04^{*}$ \\
\hline$d l f b k f$ & 0.442 & 0.401 & 0.109 & 0.376 \\
\hline$t$ & 17.67 & 12.07 & 2.78 & 8.07 \\
\hline$d l l$ & 0.222 & 0.131 & 0.271 & 0.153 \\
\hline$t$ & 3.75 & 2.82 & 4.79 & 3.30 \\
\hline dlgpepib & 0.123 & 0.095 & 0.085 & 0.075 \\
\hline$t$ & 3.21 & 2.95 & $1.94 * *$ & $1.86 * *$ \\
\hline
\end{tabular}

*FMOLS: mínimos cuadrados ordinarios completamente modificados de Pedroni (2000) considerando cointegración homogénea. Las variables se consideran en primeras diferencias y en logaritmo. Todas las variables son significativas a $1 \%$ excepto para ** que lo son a $5 \%$.

El papel de la repatriación de las utilidades de la IED no puede subvalorarse dado que el coeficiente, reuied, es estadísticamente significativo en todas las ecuaciones. En otras palabras, los resultados sugieren que la repatriación es un factor determinante de la actividad económica en la región latinoamericana. En particular, dado que el signo encontrado es negativo, se tiene que en el largo plazo la salida de utilidades de los países latinoamericanos restringe la actividad económica limitando, entonces, los efectos positivos de la presencia de capitales extranjeros en los mercados receptores. Esto es, la hipótesis que considera a la IED integral como benéfica para la economía huésped no parece sostenerse desde este enfoque.

Adicionalmente, se observa que la elasticidad estimada de reuied es en general de baja magnitud. El mayor efecto de la repatriación se registra en el consumo final, lo que siguiere que la salida de capitales puede impactar sobre el tipo de cambio, lo que a su vez afecta el saldo comercial, y en la medida que el banco central actúe o no en el mercado cambiario, contrayendo o no el medio circulante, podría, afectar las posibilidades de consumo.

Asimismo, las decisiones estratégicas de las ETN respecto a la repatriación limitan los encadenamientos productivos, tal como señala Mortimore (2006), afectando la producción agregada e industrial. De igual manera, si bien el coeficiente es muy reducido, la no reinversión de utilidades impacta negativamente en el largo plazo en el nivel de vida en las economías en la región. 
De todo lo anterior, se extraen tres aspectos críticos. Primero, desde un punto de vista de política industrial, la repatriación es un elemento que limita la actividad productiva en el largo plazo. Por ende, el fomento de la industria debe seguir una estrategia que incorpore los capitales repatriados en la producción doméstica. Segundo, el crecimiento económico en América Latina y, aún más, el desarrollo están condicionados por las decisiones de las ETN respecto a la repatriación de utilidades. Se aprecia la vulnerabilidad de este grupo de países ante la salida de estos capitales que puede traducirse en dependencia externa. Una política que atraiga IED de calidad que incluya mayores porcentajes de reinversión contribuiría a reducir el efecto negativo. Tercero, a pesar del efecto positivo de la formación de capital humano, la cantidad y calidad de este tipo de capital no parece ser suficiente como para retener un mayor porcentaje de las utilidades obtenidas. En consecuencia, parece natural seguir programas que amplíen estos elementos del capital humano quizás a través de esquemas de coinversión pública-privada (reinversión).

Cuadro 4. Causalidad de corto plazo basada en el VECM

\begin{tabular}{|c|c|c|c|c|}
\hline & \multicolumn{4}{|c|}{ Ecuación } \\
\hline$\gamma$ & dlpib & dlpibpc & dlcf & dlipi \\
\hline dlreuied & -0.350 & -0.285 & -0.332 & -0.411 \\
\hline Valor de $p$ & 0.025 & 0.013 & 0.021 & 0.017 \\
\hline$d l f b k f$ & 0.573 & 0.402 & 0.128 & 0.334 \\
\hline Valor de $p$ & 0.018 & 0.021 & 0.024 & 0.019 \\
\hline$d l l$ & 0.225 & 0.143 & 0.227 & 0.243 \\
\hline Valor de $p$ & 0.031 & 0.025 & 0.033 & 0.029 \\
\hline dlgpepib & 0.135 & 0.148 & 0.096 & 0.108 \\
\hline Valor de $p$ & 0.008 & 0.013 & 0.019 & 0.016 \\
\hline$\omega$ & -0.331 & -0.295 & -0.280 & -0.319 \\
\hline Valor de $p$ & 0.012 & 0.011 & 0.018 & 0.014 \\
\hline F-test & 4.26 & 6.21 & 3.78 & 5.16 \\
\hline
\end{tabular}

Se presenta causalidad bidireccional para los casos pib-reuied (0.223), pib-gpepib (0.240) pibpc-I (0.196), cf-l (0.347) e ipi-fbkf (0.218).

Por otro lado, en el cuadro 4 se presentan las estimaciones del VECM. Los coeficientes de ajuste del sistema al equilibrio de largo plazo $(\omega)$ son estadísticamente significativos; lo que implica la existencia de cointegración en el sistema 
propuesto. Además, en todas las ecuaciones se tiene el signo correcto, significando que contribuyen a corregir los desequilibrios por shocks estocásticos. No obstante, la velocidad de la respuesta puede considerarse baja en las cuatro ecuaciones de interés. En promedio, se corrige sólo el 14\% de la desviación cada año.

Asimismo, los coeficientes de corto plazo son estadísticamente significativos. Destaca el hecho que estas elasticidades sean para todas las variable mayores que las de largo plazo. Esto sugiere la idea de una sobrerreacción de la actividad económica que tiende a corregirse en el largo plazo. De cualquier forma, se aprecia el efecto negativo de la repatriación en las variables económicas de interés para los países seleccionados. Estos resultados son de interés para los hacedores de política puesto que sugieren la necesidad de establecer mecanismos de retención de porcentajes mayores de las utilidades obtenidas por las ETN en estos países.

Asimismo, como hay fuerte evidencia de cointegración en el panel, se pueden estimar los coeficientes específicos para cada país. Para obtener las elasticidades individuales se sigue la metodología estimaciones FMOLS considerando cointegración heterogénea. Los resultados para las elasticidades respecto a la repatriación se muestran en el cuadro 5.

Prácticamente todas las elasticidades son estadísticamente significativas, para distintos niveles, y muestran signo negativo. Los coeficientes son menores a la unidad para los países en la muestra independientemente de la medida de actividad económica considerada. Así, se confirma el efecto negativo de la no reinversión encontrado en la estimación homogénea, aunque cuando se considera cada país individualmente para acrecentarse.

En particular, Costa Rica, México y Colombia son los más afectados por la repatriación de utilidades. Esta "fuga" de capitales afecta a la economía a través de una reducción en producción agregada, ingreso per cápita, consumo final y producción industrial. El promedio de las elasticidades significativas es mayor al 0.10 por ciento para el primer país y ligeramente menor a éste para los segundos. Por el contrario, los países menos afectados son Uruguay, Paraguay, Perú y Bolivia. En ambas situaciones la razón puede atribuirse al peso relativo de las relaciones económicas con Estados Unidos.

Se analizan dos grupos de países determinados por su posición respecto a la media del coeficiente estimado. Costa Rica es el país que reporta la mayor contracción del PIB agregado y per cápita ante la salida de utilidades (por arriba de la media); sin embargo, muestra menores elasticidades del consumo y producción industrial (por debajo). En cambio, el consumo final en Panamá es el de más 
94 ECONOMÍA: TEORÍA Y PRÁCTICA • Nueva Época, número 43, julio-diciembre 2015

\section{Cuadro 5. Estimaciones mediante FMOLS (within dimension)}

\begin{tabular}{|c|c|c|c|c|c|c|c|c|}
\hline \multicolumn{9}{|c|}{ Elasticidades respecto a la reuied } \\
\hline & pib & pibpc & $c f$ & ipi & pib & pibpc & $c f$ & ipi \\
\hline \multirow[t]{2}{*}{ Argentina } & $-0.0808 * *$ & $-0.089 * *$ & $-0.056 * *$ & $-0.0131 * * *$ & -0.081 & -0.089 & -0.056 & -0.013 \\
\hline & {$[2.81]$} & [3.12] & {$[3.21]$} & {$[2.64]$} & {$[2.81]$} & [3.12] & {$[3.21]$} & [2.64] \\
\hline \multirow[t]{2}{*}{ Bolivia } & $-0.0452 * * *$ & $-0.033 * *$ & $-0.028 *$ & $-0.034 * *$ & -0.045 & -0.033 & -0.028 & -0.034 \\
\hline & {$[2.63]$} & {$[2.95]$} & {$[6.14]$} & {$[2.80]$} & [2.63] & [2.95] & [6.14] & [2.80] \\
\hline \multirow[t]{2}{*}{ Brasil } & $-0.0356 * *$ & $-0.056^{*}$ & $-0.014 * * *$ & $-0.045 * * *$ & -0.036 & -0.056 & -0.014 & -0.045 \\
\hline & [2.93] & [3.59] & [2.69] & {$[2.65]$} & [2.93] & [3.59] & [2.69] & {$[2.65]$} \\
\hline \multirow[t]{2}{*}{ Chile } & $-0.054^{* *}$ & $-0.117^{\star *}$ & -0.021 * & $-0.074^{* *}$ & -0.054 & -0.117 & -0.021 & -0.074 \\
\hline & [3.12] & [2.99] & {$[4.08]$} & [3.15] & [3.12] & [2.99] & [4.08] & [3.15] \\
\hline \multirow[t]{2}{*}{ Colombia } & $-0.1109 * * *$ & -0.159 & $-0.078 * *$ & $-0.022 * * *$ & -0.111 & -0.159 & -0.078 & -0.022 \\
\hline & {$[2.71]$} & [2.11] & [3.12] & [2.49] & {$[2.71]$} & [2.11] & [3.12] & [2.49] \\
\hline \multirow[t]{2}{*}{ Costa Rica } & $-0.1484 * *$ & $-0.284 * * *$ & $-0.017^{*}$ & $-0.016^{*}$ & -0.148 & -0.284 & -0.017 & -0.016 \\
\hline & {$[2.78]$} & {$[2.54]$} & [3.95] & [6.12] & {$[2.78]$} & [2.54] & [3.95] & [6.12] \\
\hline \multirow[t]{2}{*}{ Ecuador } & $-0.0592 * * *$ & $-0.068^{*}$ & $-0.024 * * *$ & 0.009 & -0.059 & -0.068 & -0.024 & 0.009 \\
\hline & [2.51] & [3.74] & [2.71] & [1.93] & [2.51] & [3.74] & [2.71] & [1.93] \\
\hline \multirow[t]{2}{*}{ Honduras } & $0.077^{* *}$ & $0.25^{* * *}$ & $-0.030 * * *$ & $-0.0271 * * *$ & 0.077 & 0.250 & -0.030 & -0.027 \\
\hline & [2.49] & [3.08] & [2.49] & [2.55] & [2.49] & [3.08] & [2.49] & [2.55] \\
\hline \multirow[t]{2}{*}{ México } & $-0.1088^{* *}$ & $-0.171 * *$ & $-0.059 * *$ & $-0.056^{\star}$ & -0.109 & -0.171 & -0.059 & -0.056 \\
\hline & [2.91] & {$[2.76]$} & [3.22] & [4.12] & [2.91] & {$[2.76]$} & [3.22] & [4.12] \\
\hline \multirow[t]{2}{*}{ Panamá } & $-0.074 * * *$ & $-0.062 *$ & $-0.082^{*}$ & $-0.039 *$ & -0.074 & -0.062 & -0.077 & -0.039 \\
\hline & [3.12] & [5.12] & [3.75] & [5.41] & [3.12] & [5.12] & [3.75] & [5.41] \\
\hline \multirow[t]{2}{*}{ Paraguay } & $-0.0224 * * *$ & $-0.022^{*}$ & $-0.035^{*}$ & $-0.012 * * *$ & -0.022 & -0.022 & -0.035 & -0.012 \\
\hline & [2.96] & [3.39] & [4.16] & {$[2.70]$} & [2.96] & [3.39] & [4.16] & [2.70] \\
\hline \multirow[t]{2}{*}{ Perú } & $-0.031 * * *$ & $-0.018^{* * *}$ & $-0.048^{* * *}$ & $-0.029 * * *$ & -0.031 & -0.018 & -0.048 & -0.029 \\
\hline & [2.49] & [2.84] & [3.21] & {$[2.78]$} & [2.49] & [2.84] & [3.21] & [2.78] \\
\hline \multirow[t]{2}{*}{ Uruguay } & $-0.0168^{*}$ & $-0.034^{*}$ & $-0.032 * * *$ & $-0.009 * * *$ & -0.017 & -0.034 & -0.032 & -0.009 \\
\hline & [3.58] & [3.73] & {$[2.51]$} & [3.28] & [3.58] & [3.73] & [2.51] & [3.28] \\
\hline \multirow[t]{2}{*}{ Venezuela } & -0.060 & $-0.073 * * *$ & $-0.028 * * *$ & -0.012 & -0.060 & -0.073 & -0.028 & -0.012 \\
\hline & [2.17] & {$[2.80]$} & [2.57] & [1.84] & [2.17] & {$[2.80]$} & [2.57] & [1.84] \\
\hline
\end{tabular}

FMOLS: mínimos cuadrados ordinarios completamente modificados de Pedroni (2000) considerando cointegración homogénea. Las variables se consideran en primeras diferencias y en logaritmo. *, $* * y * * *$ significativas al $1 \%, 5 \%$ y $10 \%$, respectivamente. Errores estándar entre corchetes. 
alta elasticidad. Se identifica que la respuesta de la economía mexicana siempre está por arriba de este umbral, siendo una de las más dañadas con la salida de utilidades de la IED. De forma similar, tres elasticidades de Argentina, Colombia y Panamá están en ese rango de valores (por arriba).

Por tanto, los beneficios de la interacción de capitales de estos países con eU son difusos, aunque, vía la repatriación, tiende a limitar sus posibilidades de crecimiento. Si bien las especificidades de cada país, además de la coyuntura económica mundial, determinan las decisiones diferenciadas de repatriación, es cierto que parte de las inversiones productivas en éstos son financiadas por el capital extranjero, cuyos beneficios eventualmente se mueven en sentido inverso, por lo que el crecimiento de este grupo de países queda bloqueado o, en el mejor de los casos, condicionado a la estrategia global de las ETN respecto a los usos más eficientes de las utilidades obtenidas.

\section{Conclusiones}

En este documento se estimó el efecto de largo plazo de la repatriación de las utilidades de la IED estadounidense, sobre un conjunto de economías latinoamericanas, siguiendo una metodología de cointegración de panel. Se describieron los determinantes teóricos de la decisión de reinversión de las ETN en la economía doméstica, entre ellos: alternativas de oportunidades de inversión (factor interno a las empresas), rentabilidad de las filiales, movimientos en el tipo de cambio, estímulos gubernamentales y gobernanza corporativa.

Los resultados de las pruebas de cointegración de panel muestran que en primeras diferencias, las variables forman un sistema de panel cointegrado. Las estimaciones sugieren que la inversión en capital físico, el personal empleado, el gasto público en educación y la repatriación de utilidades son estadísticamente significativos en el largo y corto plazos y muestran el signo esperado. En particular, la variable reuied afecta negativamente la actividad económica en la región. Este resultado confirma lo señalado por Mortimore (2006) y Higginbottom (2013).

Si bien las elasticidades estimadas son pequeñas, el rol de la repatriación es crucial para la actividad productiva en América Latina, principalmente en el corto plazo. Así, la repatriación de utilidades de la IED de EU se ha constituido en una barrera al crecimiento económico en la región. Estos resultados son relevantes para la política económica, puesto que se requiere diseñar y aplicar incentivos de gran alcance para influir efectivamente en el desempeño económico de 
estos países. Así, por ejemplo, para incrementar el PIB en un punto porcentual se requiere disminuir la repatriación (aumentar la reinversión) en $2.8 \%$ anualmente.

En particular, se identificó que Costa Rica, México y Colombia son los más afectados por la repatriación de ganancias. El caso mexicano es sobresaliente puesto que las cuatro variables de actividad económica consideradas reaccionan negativamente ante la salida de utilidades obtenidas por la IED. No obstante, en términos generales los resultados permiten aceptar que la no reinversión tiene efecto nocivo en las economías de la región.

Por ende, establecer un marco de regulación que propicie competitivamente las reinversiones en las economías domésticas latinoamericanas favorecería significativamente el desempeño económico. El control de estos capitales marginales en el largo plazo debe considerar que el comportamiento de las ETN está guiado por aspectos relacionados con la rentabilidad, incentivos gubernamentales directos y eficiencia de las instituciones y del capital humano, variables base del desarrollo sostenido de los países. En esencia, las medidas de política orientadas al fomento de este tipo de factores deberían retener un margen mayor de utilidades en el largo plazo. Al respecto, siguiendo a Daniels, Hejazi y Von der Ruhr (2004) la promoción gubernamental de reinversiones se justifica, desde el enfoque de las ETN, por el hecho que éstas son una de las fuentes principales de financiamiento de las ETN de Estados Unidos.

\section{REFERENCIAS BIBLIOGRÁFICAS}

Acharya, Debashis; Amanulla, S., y Joy, Sara (2009), "Financial development and economic growth in Indian states: an examination", International Research Journal of Finance and Economics, 24 (2), pp. 117-130.

Agostini, Claudio, y Jalile, Ileana (2009), "Efectos de los impuestos corporativos en la inversión extranjera en América Latina", Latin American Research Review, 44 (2), pp. 84-108.

Alfaro, Laura; Chanda, Areendam; Kalemli-Ozcan, Sebnem, y Sayek, Selin (2004), "FDI and economic growth: the role of local financial markets", Journal of International Economics, 64 (1), pp. 89-112.

Allen, Franklin; Bernardo, Antonio, y Welch, Ivo (2000), "A theory of dividends based on tax clienteles", The Journal of Finance, 55 (6), pp. 2499-2536.

Altzinger, Wilfried (2008), "The profitability of Austrian foreign direct investment-reinvestment or repatriation?”, FIw Research Reports Series 010. 
Barry, Frank. (2005), "FDI, transfer pricing and the measurement of R\&D intensity”, Research Policy, 34 (5), pp. 673-681.

Blomstrom, Magnus, y Kokko, Ari (2003), "The economics of foreign direct investment incentives", Stockholm School of Economics Working Paper 168.

Blomstrom, Magnus, y Wolff, Edward (1994), "Multinational corporations and productivity convergence in Mexico", NBER Working Paper 3141.

Blouin, Jennifer; Krull, Linda, y Robinson, Leslie (2012), "Is us multinational repatriation policy influenced by reporting incentives? The Accounting Review, 87 (5), pp. 1-19.

Borensztein, Eduardo; Gregorio, José de, y Lee, Jong-Wha (1995), "How does foreign direct investment affect economic growth?", NBER Working Paper 5057.

Brada, Josef, y Tomšík, Vladimir (2009), "The foreign direct investment financial life cycle: evidence of macroeconomic effects from transition economies", Emerging Markets Finance and Trade, 45 (3), pp. 19-34.

Breitung, Jorg (2005), "A parametric approach to the estimation of cointegration vectors in panel data", Econometric Reviews, 24 (2), pp. 151-173.

Breitung, Jorg, y Pesaran, M. Haasxhem (2005), "Unit Roots and Cointegration in Panels”, cesifo Working Paper Series 1565.

Carrillo, Jorge, y Gomis, Redi (2011), "Firmas multinacionales en México. Un primer mapeo", Frontera Norte, 46 (23), pp. 35-60.

CEPAL (2014), Foreign Investment in Latin America and The Caribbean, Santiago de Chile, Comisión Económica para América Latina y El Caribe.

Clausing, Kimberly (2001), "The impact of transfer pricing on intrafirm trade", en J. Hines (ed.), International Taxation and Multinational Activity, s.l., University of Chicago Press.

Chakrabarti, Avik (2001), "The determinants of foreign direct investment: sensitivity analyses of cross-country regressions", Kyklos, 54 (1), pp. 91-102.

Chandra, Purna, y Sahoo, Pravakar (2007), "Export-led growth in South Asia: a panel cointegration analysis", International Economic Journal, 21 (2), pp. 155-175.

Choi, In (2001), "Unit root tests for panel data", Journal of International Money and Finance, 20 (1), pp. 249-272.

Daniels, Joseph; Hejazi, Walid y Ruhr, Marc von der (2004), "Regional vs global financing strategies for US MNE", en Rugman, Alan (ed.), North American Economic and Financial Integration: Research in Global Strategic Management, vol. 10, s.l., Oxford University Press.

Desai, Mihir; Foley, Fritz, y Hines, James. (2001), "Repatriation taxes and dividend distortions”, National Tax Journal, 54:4, pp. 829-852. 
(2002), "Dividend policy inside the firm", NBER Working Paper 8698.

Dunning, John (1993), Multinational enterprises and the global economy, s.l., Addison Wesley.

Dussel, Enrique (1997), La economía de la polarización. Teoría y evolución del cambio estructural de las manufacturas mexicanas, Jus/UnAm.

Dussel, Enrique; Galindo, Luis, y Loría, Eduardo (2003), Condiciones y efectos de la inversión extranjera directa y del proceso de integración regional en México durante los años noventa: Una perspectiva microeconómica, BID/UNAM.

Echavarría, Juan (2005), "Impuestos a las utilidades e inversión extranjera directa en Colombia”, documento de trabajo del Banco de la República 025.

Engle, Robert, y Granger, Clive (1987), "Cointegration and error correction: representation, estimation and testing", Econometrica, 55 (2), pp. 251-276.

Fritsch, Winston y Franco, Gustavo (1991), "Inversión extranjera directa y pautas de la industrialización y el comercio exterior en los países en desarrollo: Notas con referencia a la experiencia brasileña", Desarrollo Económico, 30 (120), pp. 523-547.

Gligo, Nicolo (2007), "Políticas activas para atraer inversión extranjera directa en América Latina y el Caribe", Serie Desarrollo Productivo 175, CEPAL.

Granato, Leonardo, y Oddone, Carlos (2007), "La protección internacional del inversos extranjero a través de los acuerdos bilaterales de inversión”, Tendencias, 8 (2), pp. 43-66.

Groen, Jan, y Kleibergen, Frank. (2003), "Likelihood-based cointegration analysis in panels of vector error correction models", Journal of Business and Economic Statistics, 21 (1), pp. 295-318.

Gropp, Reint, y Kostial, Kristina (2000), "The disappearing tax base: is foreign direct investment eroding corporate income taxes?”, European Central Bank Working Paper 31.

Graham, John; Hanlon, Michelle, y Shevlin, Terry (2011), "Real effects of accounting rules: evidence from multinational firms' investment location and profit repatriation decisions", Journal of Accounting Research, 49 (1), pp. 137-185.

Hall, Peter, y Soskice, David (2001), "An introduction to varieties of capitalism”, en P. Hall y D. Soskice (eds), Varieties of capitalism: the institutional foundations of comparative advantage, s.l., Oxford University Press.

Higginbottom, Andy (2013), "The political economy of foreign investment in Latin America dependency revisited", Latin American Perspectives, 30 (20), pp. $1-23$.

Hines, James, y Hubbard, Glenn (1990), “Coming home to America: dividend repatria- 
tions by us multinationals", en A. Razin y J. Slemrod (eds.), Taxation in the global economy, s.l., University of Chicago Press.

Ibarra-Caton, Marilyn, y Mataloni, Raymond (2014), Direct investment positions for 2013. Country and industry detail, Bureau of Economic Analysis [pdf]. Consultado el 20 de febrero de 2015, en: http://www.bea.gov/scb/pdf/2014/07\%20 July/0714_direct_investment_positions.pdf.

Ishii, Yasunori (2006), "Multinational firms and strategic FDI subsidies", Review of International Economics, 4 (2), pp. 292-305.

Kao, Chihwa (1999), "Spurious regression and residual based tests for cointegration in panel data", Journal of Econometrics, 90 (1), pp. 1-44.

Kliman, Andrew (2012), "The falling profitability of us multinational corporations abroad. Implications for an understanding of global profitability and the Great Recession”, Marxism 21, 6 (1), pp. 218-255.

Kok, Recep, y Acikgoz, Bernur (2009), "Analyses of FDI determinants in developing countries", International Journal of Social Economics, 36 (2), pp. 105-123;

Larsson, Rolf; Lyhagen, Johan, y Löthgren, Mickael (2001), "Likelihood-based cointegration tests in heterogeneous panels", Econometrics Journal, 4 (1), pp. 109-142.

Lehmann, Alexander (2002), "Foreign direct investment in emerging markets: Income, repatriations and financial vulnerabilities", IMF Working Paper 02/47.

Lehmann, Alexander, y Mody, Ashoka. (2004), "International dividend repatriations", IMF Working Paper 04/5.

Lipsey, Robert (2002), "Home and host effects of FDI”, NBER Working Paper 9293.

Love, James, y Lage-Hidalgo, Francisco (2000), "Analysing the determinants of us direct investment in Mexico", Applied Economics, 32 (1), pp. 1259-1267.

Lucas, Robert (1993), "On the determinants of direct foreign investment: evidence from east and South Asia", World Development, 21 (3), pp. 391-406.

Lundan, Sasrianna (2006), "Reinvested profitability as a component of FDI: an analytical review of the determinants on reinvestment", Transnational Corporations, 15 (3), pp. 33-64.

Markusen, James, y Venables, Anthony (1999), "Foreign direct investment as a catalyst for industrial development", European Economic Review, 43 (1), pp. 335-356.

McCoskey, Suzanne, y Kao, Chihwa (1998), "A residual-based test of the null of cointegration in panel data", Econometric Reviews, 17 (2), pp. 57-84.

Mencinger, Joze (2003), "Does foreign direct investment always enhance economic growth?”, Kilkos, 56 (4), pp. 491-508.

Morales, Josefina. (2010), "Inversión extranjera directa y desarrollo en América Latina", Problemas del Desarrollo, 41 (163), pp. 141-156. 
Morales, Lorena; Guerrero, Patricia, y López, Mauricio. (2009), “Una evaluación de las políticas promocionales de inversión extranjera directa en América Latina”, Lecturas de Economía, 71:2, pp. 141-168.

Mortimore, Michael (2006), Globalización y empresas transnacionales: ¿Oportunidades para el desarrollo?, Santiago de Chile, CEPAL.

- (2009), "Arbitraje internacional basado en cláusulas de solución de controversias entre los inversionistas y el Estado en acuerdos internacionales de inversión: desafíos para América Latina y el Caribe”, Serie Desarrollo Productivo CEPAL 188.

Obersteiner, Erich (1973), "A decision model for the allocation of foreign funds to dividend remittances and foreign investment in a multinational corporate system with wholly-owned foreign manufacturing subsidiaries", The Journal of Finance, 28 (1), pp. 221-222.

Pedroni, Peter (1995), "Panel cointegration; asymptotic and finite sample properties of pooled time series tests, with an application to the PPP hypothesis", Indiana University Working Papers in Economics 95-013.

_ (1997), "Panel cointegration; asymptotic and finite sample properties of pooled time series tests, with an application to the PPP hypothesis: new results", Indiana University Working Paper 97-04.

(1999), "Critical values for cointegration tests in heterogeneous panels with multiple regressors", Oxford Bulletin of Economics and Statistics, 61 (1), pp. 653-670.

_ (2000) "Fully modified oLs for heterogeneous cointegrated panels", en B. Baltagi (ed.), Nonstationary panels, panel cointegration, and dynamic panels, s.l., Elsevier.

Pelegrín, Ángels (2002), “Inversión extranjera directa”, Papeles de Economía Española, 0 (93), pp. 122-134.

Phillips, Peter, y Hansen, Bruce (1990), "Statistical inference in instrumental variables regressions with I(1) processes", Review of Economic Studies, 57 (2), pp. 99125.

Rodas, Mauricio (2005), "Los regímenes de la inversión extranjera directa y sus regulaciones ambientales en México y Chile", Serie Estudios y Perspectivas CEPAL 27, pp. 69.

Romer, Paul (1990), "Endogenous technological change”, Journal of Political Economy, 98 (95), pp. 71-102.

Rugman, Alan, y Verbeke, Alain (2001), "Subsidiary specific advantages in multinational enterprises", Strategic Management Journal, 22 (3), pp. 237-250. 
Shatz, Howard, y Venables, Anthony (2000), "The geography of international investment", World Bank Policy Research Working Paper 2338.

Slaughter, Matthew (2003), "Host-country determinants of us foreign direct investment into Europe", en H. Herrmann y R. Lipsey (eds.), Foreign direct investment in the real and financial sector of industrial countries, s.1.. Springer.

UNCTAD (2013), World Investment Report 2013: Global value chains. Investments and trade for development, Suiza, UNCTAD. 